\title{
Sacral Tuberculosis: An Atypical Manifestation
}

Faisal Amir Si Mirah¹, Ahmad Faizal Roslan", Ed Simor Khan Mor Japar Khan, Rajandra Kumar Karupiah ${ }^{1}$, Zamzuri Zakaria @ Mohamad ${ }^{1}$

${ }^{1}$ Department of Orthopaedics, Traumatology \& Rehabilitation, Kulliyyah of Medicine, International Islamic University of Malaysia

Presenter: Faisal Amir Si Mirah

Sacral tuberculosis (TB) is extremely rare and its unusual entity might delay the diagnosis and treatment of this treatable disease. A 38-year-old lady presented with a 1 year history of lower back pain with radiculopathy more to right lower limb. The patient was initially treated as Prolapsed Intervertebral Disc (PID), but showed no improvement despite regular physiotherapy and medication. Subsequently, the pain was confined to the right gluteal area and became more severe. Quality of life was impaired in which patient started using walking aids and stopped working. There was a weight loss of $20 \mathrm{~kg}$. No other symptom of TB infection or history of contact with TB patient. Bowel and urinary functions were normal. Examination showed localized tenderness at the right gluteal area. Neurological assessment of both lower limbs were MRC grade 5. Blood investigations were normal including the ESR level $(17 \mathrm{~mm} /$ hour $)$. Mantoux test was positive with $18 \mathrm{~mm}$ induration. MRI revealed a large rim enhancing paravertebral collection at pre-sacral space which extended into bilateral piriformis and gluteal muscles. The patient underwent CT-guided drainage of both gluteals and specimens taken to confirm the diagnosis of TB. Patient showed significant improvement clinically within 1 week after the drainage procedure and initiation of antituberculous chemotherapy. The initial presentation of this patient mimics PID due to irritation of sciatic nerve at piriformis level. However a change in the presentation and failure of conservative treatment should raise a high index of suspicion and necessitates further investigation to establish correct diagnosis hence proper treatment can be initiated. 